\title{
Paracrine Effects of FGF23 on the Heart
}

\begin{abstract}
Maren Leifheit-Nestler* and Dieter Haffner
Department of Pediatric Kidney, Liver and Metabolic Diseases, Pediatric Research Center, Hannover Medical School, Hannover, Germany
\end{abstract}

Fibroblast growth factor (FGF) 23 is a phosphaturic hormone primarily secreted by osteocytes to maintain phosphate and mineral homeostasis. In patients with and without chronic kidney disease, enhanced circulating FGF23 levels associate with pathologic cardiac remodeling, i.e., left ventricular hypertrophy $(\mathrm{LVH})$ and myocardial fibrosis and increased cardiovascular mortality. Experimental studies demonstrate that FGF23 promotes hypertrophic growth of cardiac myocytes via FGF receptor 4-dependent activation of phospholipase $\mathrm{C} \gamma /$ calcineurin/nuclear factor of activated $\mathrm{T}$ cell signaling independent of its co-receptor klotho. Recent studies indicate that FGF23 is also expressed in the heart, and markedly enhanced in various clinical and experimental settings of cardiac remodeling and heart failure independent of preserved or reduced renal function. On a cellular level, FGF23 is expressed in cardiac myocytes and in other non-cardiac myocytes, including cardiac fibroblasts, vascular smooth muscle and endothelial cells in coronary arteries, and in inflammatory macrophages. Current data suggest that secreted by cardiac myocytes, FGF23 can stimulate pro-fibrotic factors in myocytes to induce fibrosis-related pathways in fibroblasts and consequently cardiac fibrosis in a paracrine manner. While acting on cardiac myocytes, FGF23 directly induces pro-hypertrophic genes and promotes the progression of LVH in an autocrine and paracrine fashion. Thus, enhanced FGF23 may promote cardiac injury in various clinical settings not only by endocrine but also via paracrine/autocrine mechanisms. In this review, we discuss recent clinical and experimental data regarding molecular mechanisms of FGF23's paracrine action on the heart with respect to pathological cardiac remodeling.

Keywords: fibroblast growth factor 23, cardiac remodeling, left ventricular hypertrophy, cardiac fibrosis, endothelial dysfunction, autocrine, paracrine

\section{FIBROBLAST GROWTH FACTORS (FGFS) AND THEIR RECEPTORS}

The FGF family consists of 22 members classified into seven subfamilies due to their phylogenetic relationship (1). Based on their mechanism of function, FGFs are further divided into paracrine, endocrine, and intracrine FGFs (Table 1) (2-5). Paracrine FGFs exhibit a heparan sulfate-binding site at their C-terminal region and bind to FGF receptors (FGFR) on the cell surface using heparan sulfate as a co-factor to mediate local biological activities (6). In contrast to paracrine FGFs, endocrine FGFs display a klotho-binding site at their C-termini, and due to their very low heparan sulfate-binding affinity, endocrine FGFs are directly secreted into the blood and exert their functions as endocrine hormones in various target tissues using $\alpha$-klotho or $\beta$-klotho as co-receptors for 
TABLE 1 | Classification of fibroblast growth factor (FGF) family members according to their mechanistic function.

\begin{tabular}{ll}
\hline Function & FGF \\
\hline Paracrine & FGF1, FGF2, FGF3, FGF4, FGF5, FGF6, FGF7, FGF8, FGF9, \\
& FGF10, FGF16, FGF17, FGF18, FGF20, FGF22 \\
Endocrine & FGF15/19, FGF21, FGF23 \\
Intracrine & FGF11, FGF12, FGF13, FGF14
\end{tabular}

Bold printed FGFs play a role in heart development, health, or disease conditions.

FGFRs (7-10). Intracrine FGFs are not secreted and exert their biological functions within the same cell (11). While not all FGFs are endogenously expressed in the heart of humans and rodents (12), it was shown that FGF2, FGF3, FGF8, FGF9, FGF10, FGF16, FGF15/19, FGF21, and FGF23 all act on the heart in a paracrine or endocrine manner to induce physiological or pathological pathways in heart development, health, and disease (Table 1) $(5,13)$. The specific role of these FGFs and their respective FGFRs in the heart are nicely discussed in more detail in two reviews by Itoh and colleagues $(5,13)$.

In mammals, due to alternative splicing, seven FGFRs are generated from four different FGFR genes (FGFR1, FGFR2, FGFR3, FGFR4) with different FGF-binding affinity (14). Paracrine FGFs bind heparan sulfate to promote an FGF/FGFR/heparan sulfate complex while endocrine FGFs bind $\alpha$-klotho or $\beta$-klotho to induce a complex consisting of FGF/FGFR/klotho, which both directly phosphorylate the FGFR intracellular tyrosine kinase domain. After FGFR activation, FGFR substrate $2 \alpha$ (FRS2 $\alpha$ ) is activated downstream, thereby inducing rat sarcoma protein (RAS)mitogen-activated protein kinase (MAPK), phosphoinositide 3-kinase (PI3K)-AKT serine/threonine kinase (AKT), or signal transducer and activator of transcription (STAT) signaling within the cell, respectively $(3,4,15,16)$. In addition, phospholipase $\mathrm{C} \gamma$ (PLC $\gamma$ ) can also be phosphorylated due to FGFR activation leading to activation of calcium-dependent signaling mediating cell motility (15). In the cardiovascular system, FGFR1c, FGFR2b, FGFR2c, FGFR3c, and $\beta$-klotho are physiologically expressed at very high levels while FGFR1b, FGFR3b, FGFR4, and $\alpha$-klotho are only moderately expressed (12).

\section{FIBROBLAST GROWTH FACTOR 23}

FGF23 belongs to the endocrine FGF family and mainly functions as a hormone-like FGF. It is mainly expressed and secreted by osteocytes in the bone as a $32-\mathrm{kDa}$ phosphaturic and calcitriol inhibitory protein regulating phosphate homeostasis (17). FGF23 exists in a full-length biologically active form and can be cleaved into $\mathrm{N}$ - and C-terminal fragments. The classic target organs of FGF23 are the kidney and the parathyroid glands where it exerts its physiological function through FGFRs with $\alpha$-klotho as a cofactor $(9,18)$. The FGFR-binding site is located in the $\mathrm{N}$-terminus while the $\alpha$-klotho binding site is located in the $\mathrm{C}$-terminus. The proprotein convertase cleavage site is stabilized by O-linked glycosylation via Galnt3 (19). FGF23 acts on the kidney via FGFR1c/ $\alpha$-klotho/MAPK signaling to regulate phosphate and vitamin $\mathrm{D}$ metabolism. It reduces renal phosphate reabsorption by suppression of sodium phosphate co-transporters $\mathrm{NaPi}-2 \mathrm{a}$ and $\mathrm{NaPi}-2 \mathrm{c}$, thereby lowering serum phosphate levels $(17,20)$. Moreover, FGF23 decreases active vitamin D synthesis by downregulation of $1 \alpha$-hypdroxylase and upregulation of 24-hydroxylase resulting in low levels of $1,25(\mathrm{OH})_{2} \mathrm{D}_{3}$ in serum (17). In the parathyroid gland, FGF23 inhibits the secretion of parathyroid hormone (21).

FGF23 expression is almost absent in the central nervous system, endocrine non-reproductive system, and metabolic system, and only minimally expressed in the gastrointestinal system, immune system, reproductive system, and cardiovascular system in healthy adults (12). In pathological conditions, FGF23 was shown to be excessively enhanced in bone (22), heart (23-26), liver (27), and kidneys (28, 29-32).

\section{FGF23 AND THE CARDIOVASCULAR SYSTEM}

Pathological cardiac remodeling, i.e., left ventricular hypertrophy $(\mathrm{LVH})$, myocardial fibrosis, and vascular calcification are the major cardiovascular pathologies in the general population and in patients with chronic kidney disease (CKD) presenting with $15-21 \%$ and up to $90 \% \mathrm{LVH}$, respectively (33). Since $\alpha$-klotho as a specific co-receptor for FGF23 is not expressed in human and rodent hearts $(12,25,34)$, direct effects of endocrine-acting FGF23 on the cardiovascular system were not supposed for a long time. However, the causes of cardiovascular disease pathologies are multifactorial. In 2008, FGF23 was discussed for the first time as a new mediator for the progression of LVH in CKD (35). In recent years, recent clinical and experimental studies demonstrated positive associations between endocrine-acting FGF23, cardiac remodeling, and endothelial dysfunction in pathological conditions in humans and rodents. In 2011, Faul and colleagues presented for the first time that administration of FGF23 directly induces hypertrophic growth of isolated neonatal rat cardiomyocytes in vitro and LVH in vivo in an FGFR-dependent but $\alpha$-klotho-independent manner (34). In contrast to the established FGF23/FGFR/ $\alpha$-klotho signaling complex primarily mediating the induction of RAS/MAPK pathway (9), FGF23/FGFR activates PLC $\gamma$ in cardiac myocytes and induces hypertrophic cell growth using calcineurin/nuclear factor of activated $\mathrm{T}$ cell (NFAT) signaling in the absence of $\alpha$-klotho (34). Interestingly, these effects were independent of blood pressure levels. Furthermore, it was shown that FGF23 increases intracellular calcium levels in cardiac myocytes in vitro and promotes contractility of murine cardiac myocytes and ventricular muscle strips ex vivo $\alpha$-klotho independently (36). Thus, the $\alpha$-klotho-independent action of FGF23 on the heart became more and more likely. In 2015, Grabner et al. identified the FGFR4 isoform as specific FGFR in the heart mediating FGF23's pro-hypertrophic action without $\alpha$-klotho (37). In recent years, the importance of the role of enhanced circulating FGF23 on the development of cardiac remodeling was thoroughly demonstrated in patients with CKD $(34,38-43)$ and end-stage renal disease (ESRD) $(34,35,44)$. Reaching serum levels of up to 1,000 -fold higher than in healthy individuals, FGF23, and associated alterations in 
mineral metabolism, including hyperphosphatemia, hypercalcemia, secondary hyperparathyroidism, vitamin $\mathrm{D}$, and klotho deficiency, are associated with uremic cardiomyopathy, LVH, premature death, and all cause mortality in $\operatorname{CKD}(35,38,41,45-48)$.

Clinical studies further indicate that circulating levels of FGF23 are also elevated in patients with dilated cardiomyopathy, ischemic heart disease, acute decompensated and chronic heart failure (HF), atrial fibrillation, and cardiogenic shock, despite normal renal function, suggesting that enhanced cardiac synthesis of FGF23 may cause elevated circulating FGF23 levels and thereby promote cardiac injury (49-61). The endocrine action of FGF23 on the heart and other organ systems was previously described in detail (62-69).

\section{PARACRINE ACTION OF FGF23 ON THE HEART}

It was suggested that FGF23 is not just a biomarker of increased risk but also a direct inducer of cardiac injury in the settings of CKD or HF (34, 70-72). Although FGF23 was discovered in 2001 as an endocrine hormone (73), cardiac FGF23 may be upregulated in certain clinical settings and promote cardiac remodeling via autocrine and/or paracrine mechanisms.

\section{Cardiac FGF23 and LVH}

Recently, we analyzed myocardial autopsy samples of deceased patients with childhood-onset ESRD and investigated the wellestablished FGF23/FGFR4 signaling cascade mediating cardiac hypertrophy. We demonstrated that FGF23 is expressed by cardiac myocytes in humans as the full-length biologically active protein (25). Moreover, cardiac FGF23 and FGFR4 are strongly enhanced in heart tissue of CKD patients when compared to age- and sex-matched controls and both positively associate with the development of LVH. As expected, klotho is not expressed in the human heart on mRNA level. However, soluble klotho protein can be clearly detected in myocardial tissue of CKD patients by Western blot and immunohistochemical analysis. The amount of soluble klotho in myocardial tissue is found to be reduced in CKD patients, and negatively associated with the duration of ESRD, dialysis vintage, and cardiac myocyte cross-sectional area. Interestingly, cardiac expression of FGF23, FGFR4, and the amount of soluble klotho in cardiac tissue are found to be dysregulated in dialysis patients and ameliorated in patients with functioning renal allografts. Similarly, FGF23/ FGFR4-associated calcineurin/NFAT pathway and the expression of pro-hypertrophic markers are normalized after kidney transplantation. Multiple linear regression analysis points out that cardiac FGF23 expression and the amount of soluble klotho protein in cardiac tissue are independent predictors of cardiac myocyte cross-sectional area while the mode of renal replacement therapy (RRT) at time of death is found to be the only independent predictor for the induction of cardiac FGF23 synthesis. The amount of soluble klotho protein in the cardiac tissue of CKD patients correlates with the duration of ESRD and the mode of RRT at time of death, and finally, cardiac FGFR4 expression correlates with estimated glomerular filtration rate, mode of RRT at time of death, cumulative time spent on dialysis, and cardiac FGF23 expression levels. The above-mentioned findings are supported by a recent study investigating myocardial tissue of 5/6 nephrectomized $(\mathrm{Nx})$ rats, which is a well-established model of experimental uremia (26). Indeed, cardiac Fgf23 gene expression is up-regulated in myocardial tissue of $5 / 6 \mathrm{Nx}$ rats compared with sham operated animals. Furthermore, experimental uremia induces full-length cardiac Fgf23 protein demonstrated by Western blot, which positively correlates with the heart weight to body weight ratio, and enhanced $\beta M H C$ and $B N P$ expression levels. Although these association studies do not prove causality, data suggests the existence of a paracrine FGF23 mechanism in the heart mediating LVH in uremic cardiomyopathy.

Studies in patients with acute decompensated heart failure (ADHF) point out that circulating FGF23 levels are significantly enhanced in ADHF compared to controls, whereas myocardial FGF23 gene expression is not consistently altered (60). However, the investigated study cohort is rather small and FGF23 mRNA levels greatly vary within both groups, which may have biased the results of this study. Since ADHF is defined as sudden or gradual onset of HF symptoms (74), the degree of HF progression may also define the strength of cardiac FGF23 induction. Nevertheless, FGF23 is detected in myocardium of both $\mathrm{HF}$ and normal patients by quantitative real-time PCR confirming the presence of FGF23 in the human myocardium. In addition, immunohistochemical analysis detects FGF23 expression in the cardiac myocytes of normal myocardial tissue and in failing hearts.

A recent study by Slavic and colleagues investigated cardiac and circulating FGF23 in experimental heart hypertrophy (75). They showed that increased afterload due to transverse aortic constriction (TAC) in mice induces an increase in circulating intact Fgf23 serum levels within $24 \mathrm{~h}$ after TAC, which maintains for at least 4 weeks post-surgery. TAC significantly increases cardiac Fgf23 gene transcripts while bone Fgf23 production is higher overall compared to the heart but not significantly stimulated by TAC. Furthermore, pressure overload dramatically reduces the cleavage of Fgf23 in serum. Interestingly, expression of Galnt3, which $\mathrm{O}$-glycosylates and thereby stabilizes Fgf23 protein, is increased in the heart post-TAC but unchanged in bone. By contrast, expression of Fam20c and Furin, promoting Fgf23 cleavage, remain unchanged in heart tissue of mice after TAC. Taken together, these data suggest that cardiac FGF23 synthesis is induced due to pressure overload and reduced FGF23 cleavage further contributes to enhanced intact FGF23 in the heart. Importantly, the up-regulation of cardiac and serum intact Fgf23 is not due to alteration of mineral metabolism as serum levels of phosphate, calcium, sodium, potassium, and iron remain unchanged in mice after TAC compared to sham-operated animals. Instead, serum aldosterone levels, shown to stimulate FGF23 expression in vitro (76), are induced in TAC-operated mice. To confirm whether induction of renin-angiotensin-aldosterone system (RAAS) triggers pressure overload-induced up-regulation of cardiac Fgf23, the authors treated wild-type mice with spironolactone for 2 weeks post-TAC surgery (75). Administration of spironolactone inhibits the induction of circulating intact Fgf23 levels in TAC mice and reduces the mRNA expression of Fgf23 in bone but not in the heart, suggesting that a pressure overload-induced 
increase in bone Fgf23 synthesis is driven by aldosterone secretion in TAC mice. Interestingly, heart weight to body weight ratio, cardiac myocyte size, end-diastolic left ventricular wall thickness, fractional shortening, and mean blood pressure after TAC are not improved by aldosterone inhibition, indicating that increased systemic Fgf23 may be less important in the development of pressure overload-induced cardiac hypertrophy. However, TACinduced expression of cardiac Fgfr1, Fgfr3, and Fgfr4, nuclear localization of NFAT protein within the cardiac myocytes, Rcan 1 induction, and consequently cardiac hypertrophy and fibrosis are also present in global homozygous $\mathrm{Fg} f \mathrm{3}^{-/-} /$vitamin D receptor $(\mathrm{VDR})^{\Delta / \Delta}$ or $\mathrm{Klotho}^{-/-} / \mathrm{VDR}^{\Delta / \Delta}$ double knockout mice compared to respective wild-type mice. Neither deletion of Fgf23 protected against pressure overload-induced cardiac hypertrophy and fibrosis, nor klotho deficiency exacerbated TAC-induced pathological hypertrophic phenotype in mice. Thus, FGF23 would appear to be a consequence of pressure overload-induced hypertrophy and perhaps not the initial inducer, suggesting that additional factors could mediate hypertrophic responses during pressure overload independent of FGF23.

FGF2 is expressed in cardiac myocytes and fibroblasts, and angiotensin II or adrenergic stimulation induces FGF2 synthesis in cardiac myocytes, which in turn stimulates itself in a feedforward loop (77). High blood pressure induces LVH via upregulation of FGF2, which is shown to be necessary for hypertrophic development in the TAC model (78). Neonatal and adult cardiac fibroblasts mainly express the high-molecular weight isoform of FGF2, which is stimulated and secreted through angiotensin II treatment, thereby acting on cardiac myocytes via induction of a fetal gene program resulting in cardiac hypertrophy (79). However, neither spironolactone treatment nor FGF23 knockout alters the induction of FGF2 in the high blood pressureinduced cardiac hypertrophy through TAC (75). Interestingly, it was shown that high-molecular weight FGF2 isoform directly stimulates FGF23 synthesis in the bone $(80,81)$. Whether upregulation of FGF2 in the heart due to pressure overload-induced cardiac hypertrophy after TAC is also responsible for the induction of cardiac FGF23, and if so, the role of cardiac FGF23 in this setting has to be further elucidated.

However, these conclusive findings coincide with clinical and experimental studies showing that enhanced FGF23 serum levels and/or the induction of cardiac FGF23 synthesis are not associated with high blood pressure-induced $\operatorname{LVH}(25,34,37)$. In addition, the fact that cardiac FGF23 is still enhanced after mineralocorticoid receptor (MR) blockade while bone FGF23 and systemic FGF23 are reduced supports our hypothesis that induction of cardiac FGF23 may have additional blood pressureindependent paracrine effects on the heart, which needs to be addressed in future studies.

\section{Cardiac FGF23 and Myocardial Fibrosis}

The role of circulating and/or cardiac FGF23 in the progression of cardiac fibrosis and its potential effects on myocardial fibroblasts promoting cardiac diastolic function is largely unknown. Initial experimental data in rodents demonstrated that FGF23 stimulates the renal expression and production of transforming growth factor $\beta$ (TGF- $\beta$ ) $(82,83)$. TGF- $\beta$ is a classic cytokine involved in different signaling pathways, which is shown to induce fibrosis in various tissues.

Recently, Smith et al. reported that FGF23 is synthesized locally by renal tubules and activates injury-primed fibroblasts (31). Moreover, FGF23 stimulates gene expressions involved in TGF- $\beta$ signaling pathways via FGFR4-dependent activation of NFAT and enhancement of TGF- $\beta$ autoinduction in injuryprimed renal fibroblasts (84). These data suggest a paracrine mechanism of FGF23 in the kidney acting on renal fibroblasts to induce pro-fibrotic signaling pathways.

In the heart, cardiac FGF23 expression is upregulated in post-myocardial infarction (MI) in rodents and mediates cardiac fibrosis through the activation of $\beta$-catenin (24). $\beta$-catenin is a pro-fibrotic factor cross talking with TGF- $\beta$ signaling $(83,85,86)$. Hao and colleagues demonstrated that FGF23 is endogenously expressed in neonatal rat cardiac myocytes and fibroblasts, adult mouse cardiac fibroblasts (AMCF), and the adult mouse heart (24). They also confirm the findings of Slavic et al. mentioned above (75), showing an increase in cardiac FGF23 mRNA and protein levels in pressure overload-induced failing mouse hearts after TAC. In addition, FGF23 plasma levels are significantly induced post-MI compared to sham-operated mice. Angiotensin II and phenylephrine, both pro-fibrotic and pro-hypertrophic stimulators, clearly up-regulate FGF23 expression in AMCF. Furthermore, stimulation with FGF23 promotes proliferation, collagen I and III synthesis, and $\beta$-catenin activation in AMCF. Cardiac-specific overexpression of FGF23 using adeno-associated virus carrying FGF23 (AAV-FGF23) in mice followed by MI or ischemia reperfusion significantly enhances the activation of cardiac $\beta$-catenin, TGF- $\beta$, collagen I and III, and cardiac fibrosis in mice injected with AAV-FGF23 compared to negative controls. It is important to note that the injection of AAV-FGF23 does not result in enhanced $\beta$-catenin, TGF- $\beta$, or collagen I and III expression levels in sham-operated mice compared with respective control animals. Thus, endogenous cardiac FGF23 synthesis may only impair myocardial fibrosis after MI or ischemia reperfusion through induction of paracrine signaling pathways, including activation of $\beta$-catenin and TGF- $\beta$. However, the activation of $\beta$-catenin-mediated induction of TGF- $\beta$ and collagen synthesis by FGF23 under healthy conditions remains controversial.

Andrukhova and colleagues demonstrated that both skeletal and cardiac Fgf23 expression are increased in mice and rats after MI independent of changes in serum soluble klotho levels (23). In addition, they found increasing serum intact Fgf23 levels in mice and rats after experimental MI, and immunohistochemical staining of heart tissue clearly reveals expression of Fgf23 in left ventricular cardiac myocytes after $\mathrm{MI}$ in mice and rats but not in infiltrating leukocytes within the infarct scar, suggesting that cardiac myocytes are the major cellular source of increased cardiac Fgf23 expression post-MI. Importantly, despite the induction of Fgf23, serum levels of PTH, phosphate, calcium, and sodium, and urinary excretion of phosphate, calcium, and sodium remain unchanged post-MI. In addition, biochemical markers of bone metabolism, including urinary excretion of collagen cross-link deoxypyridinoline, serum alkaline phosphatase, Dickkopf-1, and osteoprotegerin, do not differ between mouse and rats post-MI compared with sham control-operated animals. Although the 
molecular link between cardiac injury and enhanced Fgf23 levels remains to be further elucidated, these data strongly support the hypothesis that cardiac FGF23 expression is linked to pathological cardiac changes and may exert paracrine mechanisms in the heart.

In an extension to experimental studies in rats and mice, we recently analyzed the myocardial tissue of CKD patients and investigated cardiac FGF23 expression and myocardial fibrosis (87). Cardiac FGF23 synthesis and accumulation of fibrillar collagen fibers are strongly enhanced in CKD patients on dialysis treatment compared to controls. Cardiac fibrosis correlates with duration of dialysis and soluble klotho deficiency detected by immunohistochemical staining of klotho protein in heart tissue sections. Interestingly, no significant correlations between cardiac FGF23 expression and fibrosis could be detected in CKD patients. By performing human fibrosis RT profiler PCR array analyses of myocardial tissue in these patients, angiotensinogen $(A G T)$, and angiotensin II-driven pathways known to promote fibrotic response, including collagen remodeling, activation of TGF- $\beta /$ TGF- $\beta$ receptor/Smad complexes, and induction of pro-hypertrophic and pro-fibrotic growth factors endothelin-1 and connective tissue growth factor (CTGF), are significantly enhanced in patients on dialysis treatment. Although cardiac fibrosis positively correlates with the induction of $A G T$ in the heart, soluble klotho deficiency but not endogenous FGF23 expression is shown to be correlated with enhanced cardiac TGF$\beta /$ TGF- $\beta$ receptor pathway. We performed further in vitro studies using neonatal rat ventricular myocytes (NRVM) and fibroblasts (NRCF) in order to discriminate which cell type express FGF23 in the heart and may induce pro-fibrotic signaling. Hereby, we could clearly detect endogenous $\mathrm{Fgf} 23 \mathrm{mRNA}$ expression in NRVM but not in NRCF, which is in contrast to Hao et al. also demonstrating FGF23 production in cardiac fibroblasts of neonatal rats and adult mice (24). However, treatment of NRCF with recombinant FGF23 significantly stimulates collagen remodeling, promotes the induction of pro-fibrotic TGF- $\beta /$ TGF- $\beta$ receptor/Smad complexes, and induces Ctgf and endothelin-1 expression in vitro (87). Importantly and in line with the Hao et al. study mentioned above, FGF23 significantly stimulates proliferation and migration of NRCF. In addition, FGF23 induces angiotensinogen expression in cardiac myocytes, promotes extracellular matrix remodeling, and induces pro-fibrotic and pro-hypertrophic growth factors in NRVM while TGF- $\beta /$ TGF- $\beta$ receptor signaling unchanged. Taken together, our data suggest that secreted by cardiac myocytes, FGF23 may directly target cardiac fibroblasts and promote profibrotic mechanisms in a paracrine manner. This mechanism in concert with its autocrine induction of fibrotic and hypertrophicrelated factors in cardiac myocytes, cardiac FGF23 may act on both cardiac cell types and thereby promote cardiac hypertrophy and myocardial fibrosis in a paracrine/autocrine fashion.

\section{Cardiac FGF23 and Endothelial Dysfunction}

Clinical and experimental studies demonstrated that high FGF23 serum levels, hyperphosphatemia, and klotho deficiency are strongly associated with cardiovascular complications in CKD patients (71). Elevated FGF23 serum levels associate with endothelial dysfunction and small vessel disease, stroke, and brain infarction in patients with CKD stage 3-4 (40) and in the general population $(58,61,88)$, respectively. Whether enhanced circulating levels of FGF23 also impact on vascular calcification is still an open question $(39,42)$. Voigt and colleagues identified FGF23 accumulation in calcified carotid atherosclerotic lesions from subjects with normal renal function (89). In addition, a recently published study by van Venrooij et al. evaluated the relationship between kidney function, vascular calcification, and FGF23 expression in patients with heart disease (90). They detected FGF23 protein expression by immunohistochemistry in coronary arteries of patients undergoing heart transplantation, which associates with declining renal function. Vascular FGF23 expression and FGFR1, FGFR3, and $\alpha$-klotho are observed in both tunica intima and tunica media in areas of calcification. Interestingly, co-immunostaining for FGF23 and CD68 demonstrates co-localization of FGF23 with inflammatory macrophages. Quantitative real-time PCR analysis confirms the gene expression of FGF23 in human coronary artery tissue, also suggesting a potential paracrine effect of FGF23 in vessels.

We performed in vitro studies using human coronary artery endothelial cells (HCAEC) in order to investigate the underlying molecular pathways of FGF23-mediated endothelial cell function in the presence and absence of its co-receptor $\alpha$-klotho (91). First, we confirmed the previously reported findings (90) that FGFR1 and $\alpha$-klotho are present in the intima of human coronary arteries. In addition, cultured HCAECs express FGFRs and $\alpha$-klotho, and FGF23 specifically stimulates phosphorylation of FGFR1 and thereby activates the receptor, suggesting that vascular endothelial cells are a target for paracrine FGF23 signaling. Although $\alpha$-klotho is not altered by FGF23 treatment on mRNA or protein level, FGF23 increases the expression of ADAM17, responsible for cleavage of membrane bound $\alpha$-klotho, and consequently the secretion of soluble $\alpha$-klotho. Investigating endothelial cell function targeted by FGF23, we showed that FGF23 activates intracellular signaling to induce the release of nitric oxide (NO) via activation of AKT/endothelial NO synthase (eNOS) pathway in vitro in the presence of $\alpha$-klotho. Furthermore, FGF23 treatment enhances the formation of reactive oxygen species (ROS) through induction of NADPH oxidase 2 (Nox2)/p67phox/p47phox/Rac1 signaling complex. In addition, FGF23 stimulates the expression of ROS detoxifying enzymes manganese superoxide dismutase and catalase by activating Foxo forkhead transcription factor $3 \mathrm{a}$ (Foxo3a) in the presence of $\alpha$-klotho, indicating that FGF23induced ROS production is being neutralized by antioxidant mechanisms. Co-treatment with FGF23 and pan-FGFR inhibitor blunts all NO synthesis, ROS formation, and ROS degradation. Taken together, these data suggest that FGF23 effects on HCAEC mediating endothelial function in the presence of $\alpha$-klotho are FGFR dependent. Inhibition of $\alpha$-klotho using a neutralizing antibody specifically blocks FGF23-mediated activation of AKT/ eNOS and consequently the release of NO. Most importantly, blocking of $\alpha$-klotho further inhibits the expression of SOD2 and catalase induced by FGF23 but does not affect FGF23 stimulated Nox2 signaling and ROS formation. Our data suggest that in states of Klotho deficiency, e.g., CKD, FGF23-induced NO synthesis is blunted and ROS formation overrules ROS degradation. 
Consequently, enhanced circulating as well as locally synthesized FGF23 may promote endothelial dysfunction and further impact on the progression of cardiovascular disease in CKD. However, the hypothesized paracrine signaling mechanism of FGF23 mediating functional response in vessels has to be further proven in experimental animal models.

\section{FGF23 and Chronic Inflammation}

Cardiac hypertrophy and myocardial fibrosis can be promoted by pro-inflammatory cytokines, such as TNF- $\alpha$ or IL- 6 , and pro-fibrotic molecules including TGF- $\beta$ and angiotensin II (92). Inflammatory markers, including IL-6, CRP, TNF- $\alpha$, and fibrinogen, are independently associated with circulating levels of FGF23 in patients with CKD, and FGF23 associates with a greater odds ratio of severe inflammation in these patients (93). Furthermore, FGF23 directly stimulates hepatic secretion of pro-inflammatory cytokines in wild-type mice and in cultured hepatocytes by activating FGFR4 independent of $\alpha$-klotho (94), indicating a novel mechanism of FGF23 and chronic inflammation. Furthermore, chronic inflammation in cardiac fibroblasts was shown to induce the expression of FGF23 in the heart (95), suggesting a paracrine signaling mechanism of chronic inflammation inducing cardiac FGF23. However, hard evidence from experimental studies is lacking.

The cytokine oncostatin M (OSM) belongs to the IL-6 family and is involved in various biological processes, including inflammation and ischemic heart disease (96). OSM is further shown to be a major mediator of cardiac remodeling (97). After binding to a receptor heterodimer consisting of gp130 and OSM receptor $\beta$, OSM induces a Janus Kinase/STAT, MAPK, or PI3K/ AKT signaling pathway, respectively (98-100), also all known to be induced by FGF/FGFR complexes. Richter and colleagues find that FGF23 is present in cardiac myocytes of patients with ischemic cardiomyopathy, myocarditis, and dilated cardiomyopathy (101). In addition, adult rat cardiac myocytes clearly expresses $F g f 23$ on the mRNA level, which is excessively induced by OSM treatment in vitro (102). Moreover, cultured OSMtreated non-cardiac myocytes, mainly fibroblasts, also synthesize FGF23. However, FGF23-expressing non-cardiac myocytes are only rarely observed in diseased human heart tissue (101). ELISA quantifications of cardiac myocyte cell supernatant demonstrates significantly increased C-term FGF23 concentrations due to OSM, and Western blot analysis revealed that OSM stimulates both unmodified full-length FGF23 protein and cleaved FGF23 fragments in cell lysates of adult rat cardiac myocytes (102). Similarly, in corresponding cell supernatant C- and N-terminal FGF23 fragments are clearly detected. Inflammatory HF in mice caused by macrophages infiltration of the myocardium shows a strong increase in FGF23 expression and secretion in cardiac myocytes, which is associated with macrophage infiltration and activation of OSM receptor $\beta$ cascade leading to cardiac myocyte remodeling with enhanced $\alpha$-smooth muscle actinin expression, STAT3 phosphorylation, induction of atrial natriuretic peptide (ANP), and destrin. These findings suggest that FGF23 is endogenously expressed in cardiac myocytes in inflammatory HF of humans and rodents and associates with macrophage infiltration and OSM receptor activation.
FGF23 is also shown to be expressed in pro-inflammatory M1 macrophages and specifically up-regulated by lipopolysaccha-

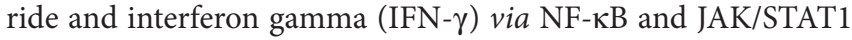
pathways, respectively (103), indicating that FGF23 produced by M1 macrophages may modulate pro-inflammatory functions in vitro and may support innate immune response to tissue injury. Therefore, it is possible that endogenous expression and secretion of FGF23 by infiltrating macrophages induce cardiovascular and kidney disease progression through induction of inflammation and fibrosis.

\section{Cardiac FGF23 and RAAS}

The activation of RAAS was shown to have direct hypertensive effects and stimulates pro-inflammatory, pro-hypertrophic, and pro-fibrotic pathways in cardiac cells, which are pharmacologically suppressed by angiotensin-converting enzyme (ACE) inhibitors, angiotensin receptor blockers (ARB), and MR antagonists (92). The initial RAAS factor is the induction of angiotensinogen expression. Renin converts angiotensinogen into angiotensin I, and angiotensin I is further metabolized to angiotensin II by ACE, finally affecting myocardial hypertrophy and fibrosis. In contrast, angiotensin-converting enzyme 2 (ACE2) degrades angiotensin I and II into angiotensin 1-9 and 1-7, respectively, acting as vasodilatory and hypotensive reagents. It was shown that FGF23 directly stimulates RAAS through inhibition of ACE2 (82) and recent studies indicate that activation of RAAS in turn can also induce FGF23 synthesis (104). In addition, renin is suppressed by $1,25\left(\mathrm{OH}_{2}\right) \mathrm{D}_{3}$ thereby avoiding RAAS activation (66). Since FGF23 suppresses $1,25\left(\mathrm{OH}_{2}\right) \mathrm{D}_{3}$ metabolism in the kidney, one may speculate that FGF23 also alters RAAS indirectly through inhibition of $1,25\left(\mathrm{OH}_{2}\right) \mathrm{D}_{3}$. However, the precise mechanisms of how cardiac FGF23 interacts with the local RAAS in the heart and thereby induce cardiac remodeling and impair diastolic function, remains to be further elucidated.

Angiotensin II and aldosterone, as active RAAS components, promote vascular and myocardial fibrosis as well as cardiac hypertrophy also independent of high blood pressure through pathologic signaling mechanisms involving cardiac myocytes and immune cells (92). However, the role of angiotensin II in health and disease is multifactorial, cardiac myocytes and fibroblasts express angiotensin II-type 1 and 2 receptors (AT1R, AT2R). Additionally, as we recently demonstrated, isolated cardiac myocytes and fibroblasts of neonatal rats clearly express angiotensinogen (Agt), which was affected by FGF23 treatment in vitro, although Agt mRNA levels are frequently higher in cardiac myocytes than in fibroblasts (87). In addition, pro-fibrotic and pro-hypertrophic molecules, i.e., TGF- $\beta$, collagen 1 , CTGF, and endothelin-1, known to be induced by AngII and/or aldosterone, are stimulated by FGF23 treatment in both cardiac myocytes and fibroblasts. Importantly, both AngII and aldosterone significantly up-regulate endogenous FGF23 expression in cardiac myocytes and induce hypertrophic cell growth, $\beta M H C, A N P$, and $B N P$. Whether these pathologies promoting cardiac hypertrophy and fibrosis are directly due to RAAS-mediated up-regulation of FGF23 in the heart or indirectly through the FGF23-mediated stimulation of RAAS is not clear. However, cardiac myocytes and fibroblasts may be potent target cells for local, non-canonical 
RAAS activation and paracrine FGF23 signaling (Figure 1). Taken these findings together, RAAS is an important regulator and key player in cardiovascular remodeling, resulting in HF that promotes sympathetic activation, systemic inflammation, and pathological cardiac hypertrophy. In turn, AngII and aldosterone induce FGF23 expression in cardiac myocytes in a feed-forward loop, although the underlying molecular mechanisms remain to be identified.

\section{FUTURE PERSPECTIVES AND POTENTIAL THERAPEUTIC OPTIONS TO PREVENT FGF23-ASSSOCIATED CARDIOVASCULAR PATHOLOGIES}

The possibilities of a therapeutic intervention to reduce or even prevent FGF23-associated cardiovascular diseases are certainly more diverse. Direct targeting of the FGF23/FGFR signaling complex is theoretically a straightforward option. It was shown that treatment with a monoclonal anti-FGF23 neutralizing antibody improved secondary hyperparathyroidism with increased vitamin D synthesis and normalization of bone markers in a rat model of experimental uremia (105). Nevertheless, chronic inhibition of FGF23 resulted in increased calcium and phosphate load, enhanced aortic calcification, and higher mortality of CKD rats compared to controls. In contrast, anti-FGF23 antibody therapy was proven to be effective and safe to cure bone abnormalities in hereditary causes of elevated FGF23 levels but reduced serum phosphate levels, e.g., X-linked hypophosphatemic rickets (106). Taken together, in states of high phosphate, general blocking of FGF23 is not suitable. Regarding the heart, the physiological relevance of locally produced FGF23 in the heart and the specific (side) effects of cardiac FGF23 suppression in health and disease are yet not clear and has to be elucidated in the future.

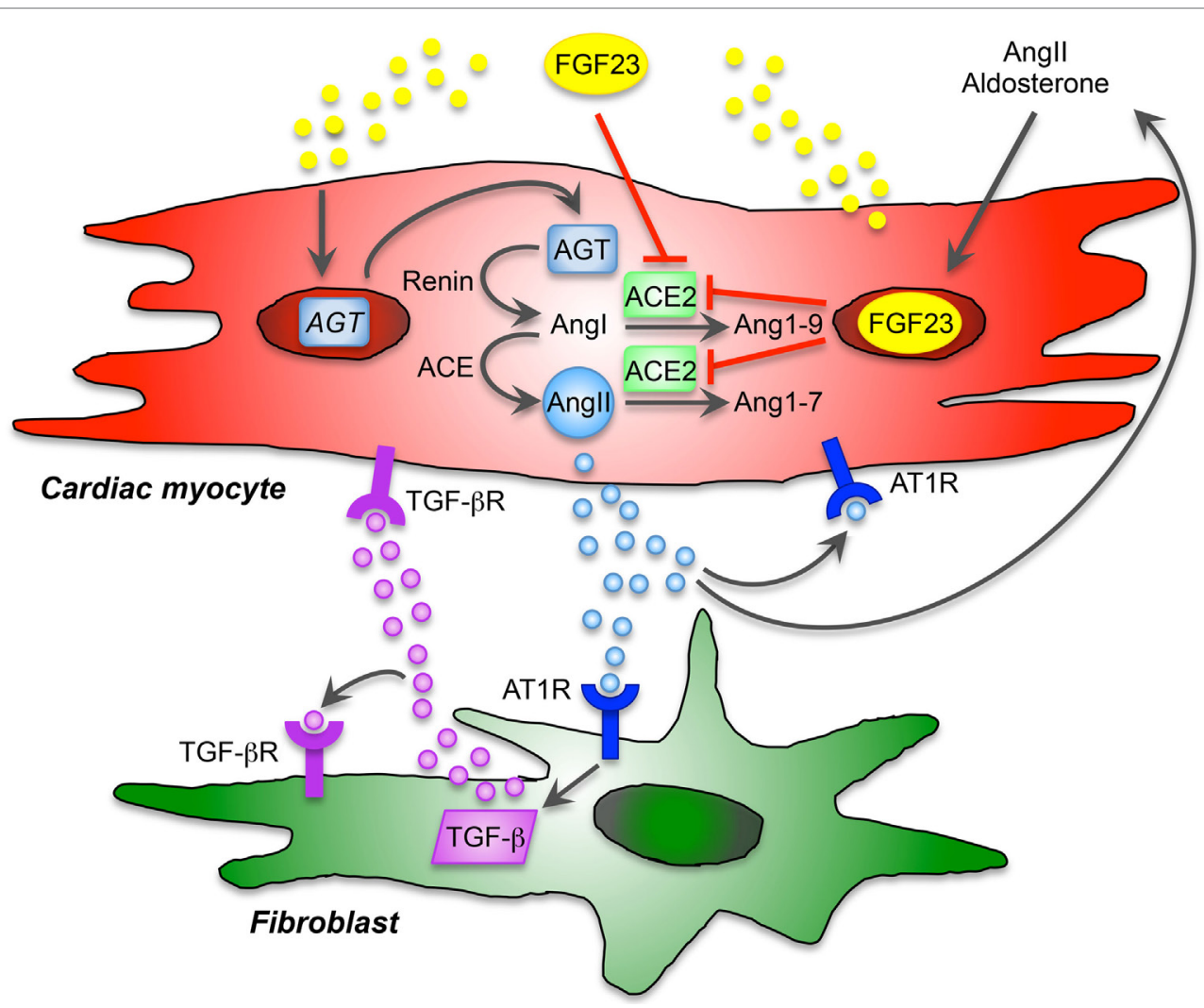

FIGURE 1 | Proposed model of cardiac FGF23 and local renin-angiotensin-aldosterone system (RAAS) in the heart. The local, non-canonical activation of RAAS directly contributes to cardiac remodeling and hypertrophy and affects different cardiac cell types. Angiotensin II (Angll) type 1 receptor (AT1R) is expressed by cardiac myocytes and fibroblasts and thereby displays an important role in maladaptive remodeling. Angll and aldosterone are stimulators of FGF23 expression and secretion, and cardiac FGF23 directly induces the expression of angiotensinogen (AGT) mRNA in cardiac myocytes. After activation, AGT is converted into Angl by renin and further to Angll through angiotensin-converting enzyme (ACE). Angiotensin-converting enzyme 2 (ACE2), which physiologically cleaves Angl and Angll counteracting the pathological effects of Angll, is inhibited by FGF23. Thus, FGF23 directly contributes to RAAS activation via induction of AGT and suppression of ACE2 in cardiac myocytes resulting in enhanced Angll and aldosterone synthesis. Angll binds to AT1R and promotes hypertrophic response in cardiac myocytes in an autocrine/paracrine manner and stimulates cardiac FGF23 in a feed-forward loop. In addition, FGF23-mediated activation of RAAS and consequently increased Angll contribute to cardiac fibrosis via binding of Angll to AT1R on cardiac fibroblasts, which directly promotes differentiation and matrix production in cardiac fibroblasts. In addition, Angll/AT1R induces the expression of transforming growth factor $\beta$ (TGF- $\beta$ ), which in turns binds to TGF- $\beta$ receptor (TGF- $\beta$ R) on cardiac fibroblasts to induce itself or stimulate hypertrophic molecules and signaling in cardiac myocytes. Taken together, endogenous expression of FGF23 in cardiac myocytes is directly involved in the activation of local RAAS contributing in pathological hypertrophy and fibrosis in a paracrine manner. 
Targeting specific FGFRs may be another efficient strategy for therapeutic interventions with lesser side effects. An overview of FGFR small-molecule compounds, monoclonal antibodies, and FGFR analogs currently under development for the use as pro- or anti-FGF signaling therapeutics among others for the treatment of cardiovascular diseases in humans, is nicely summarized in a Review by Katoh (107). Regarding the treatment of LVH, Grabner and colleagues impressively demonstrated that targeting FGFR4 specifically prevents FGF23-induced cardiac hypertrophy in vitro and in vivo in CKD and non-CKD models (37). However, the specific FGFRs mediating the FGF23-induced signaling pathways in cardiac fibroblasts and other non-cardiac myocyte cells, such as endothelial cells and macrophages, is not finally discovered so fare. Moreover, whether blocking of FGFR4 also ameliorates cardiac fibrosis, improve endothelial function or reduce inflammatory processes is not known. In addition, the translation of

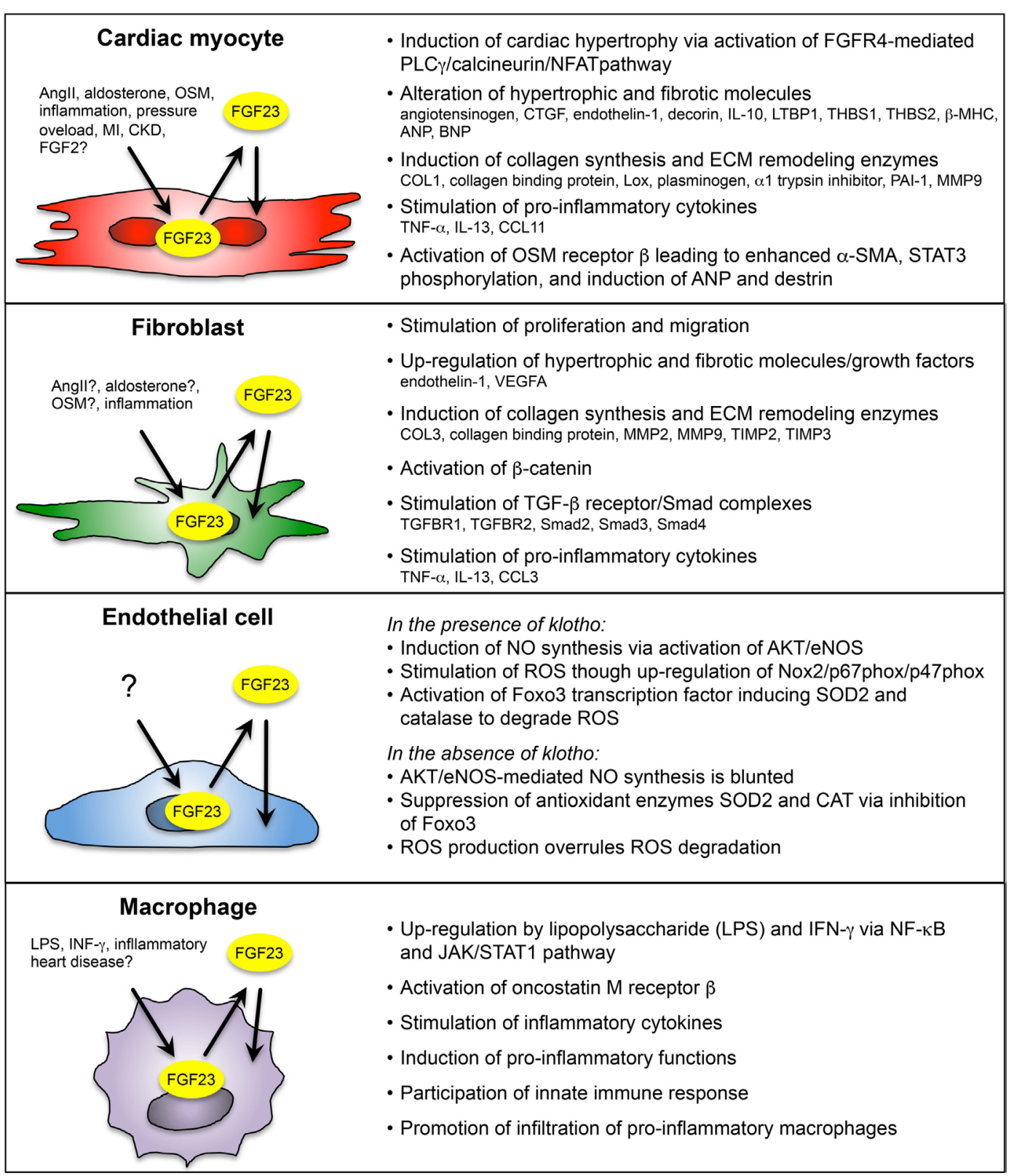

FIGURE 2 | Overview of paracrine/autocrine acting FGF23 on cardiac myocyte and non-cardiac myocyte during cardiac remodeling and heart failure (HF). FGF23 is endogenously expressed in cardiac myocytes, fibroblasts, coronary artery endothelial cells, and macrophages in the heart. Depending on the cell type, FGF23 is induced by various pathogenic stimuli, including angiotensin II (Angll), aldosterone, oncostatin M (OSM), interferon $\gamma$ (IFN- $\gamma$ ), lipopolysaccharide (LPS), inflammation, pressure overload, myocardial infarction (MI), and chronic kidney disease (CKD). Cardiac FGF23 exerts pro-hypertrophic, pro-fibrotic, and pro-inflammatory response in different cardiac cell types, which in turn activates pathologically phenotypic changes in the same or nearby cells through stimulation and secretion of growth factors, inflammatory cytokines, and pro-hypertrophic and pro-fibrotic molecules. [References for FGF23 in cardiac myocytes (23, 25, 26, 34, 37, 87, 102); cardiac fibroblasts $(24,87,101)$; endothelial cells $(90,91)$; and macrophages $(90,95,103)$.] 
this approach into clinical settings has to be investigated in future clinical studies.

The involvement of FGF23 in the RAAS activation is of particular interest and might be another promising target pathway for the therapeutic intervention of FGF23-mediated cardiac pathology. In patients with stable ischemic heart disease (SIHD) within the Prevention of Events With AngiotensinConverting Enzyme (PEACE) trial, high FGF23 levels were independently associated with risk of HF or cardiovascular mortality (108). Furthermore, ACE inhibitor (ACEi) therapy reduced the incident of both events only in the patient subgroup with the highest FGF23 quartile. A clinical study in patients with advanced chronic systolic HF showed that the absence of ACEi therapy independently associated with increased circulating FGF23 levels (59). Interestingly, in this study, ACEi therapy was further associated with a lower risk of death, urgent heart transplantation, and ventricular assist device implantation only in HF patients without CKD in the highest FGF23 tertile, but not in those with CKD. The authors conclude that the association of FGF23 with adverse events reflects the activation of RAAS. In a recently published large multicentric European prospective observational study in patients with new onset and worsening HF (BIOSTAT-CHF trail), patients with the highest FGF23 quintile presented with worse renal function, more severe HF, increased congestion, and enhanced renin and aldosterone levels suggesting pronounced RAAS activation (109). Furthermore, higher plasma levels of FGF23 were independently associated with higher aldosterone levels and less successful uptitration of ACEi and ARB in addition to increased risk of mortality and HF hospitalization. In contrast, the use of beta-blocker was not significantly associated with high FGF23 concentrations. In CKD patients, it was shown that high FGF23 levels correlated with impaired ACE inhibition (110). In addition, CKD patients with the highest FGF23 responded with less effective ACEi therapy.

However, all of these studies hypothesized that increased FGF23 levels may provide additional information regarding the tolerability and effectiveness of RAAS blockade and may identify a subset of patients with SIHD or HF with or without CKD which may benefiting from an ACEi therapy. Nevertheless, only the effects of systemic RAAS inhibition on circulating FGF23 levels were investigated and very little is known about the impact of the local RAAS/FGF23 system in the heart. It is unknown if systemic treatment with RAAS inhibitors effectively target cardiac FGF23. A small cohort study in pediatric CKD patients on dialysis treatment or with functioning renal allograft failed to associate the expression of cardiac FGF23 with RAAS medications (25). One can speculate that the impaired efficiency of ACEi therapy in renal insufficiency is caused by additional overexpression of FGF23 in the heart, which may be resistant to systemic RAAS inhibition. This hypothesis is supported by the recently reported interactions of cardiac FGF23 synthesis and RAAS (87). AngII and aldosterone induce endogenous FGF23 in the heart and FGF23 stimulates $A G T$ expression as well as the activation of RAAS in a feed forward loop, which may result in a resistance of therapeutic intervention.

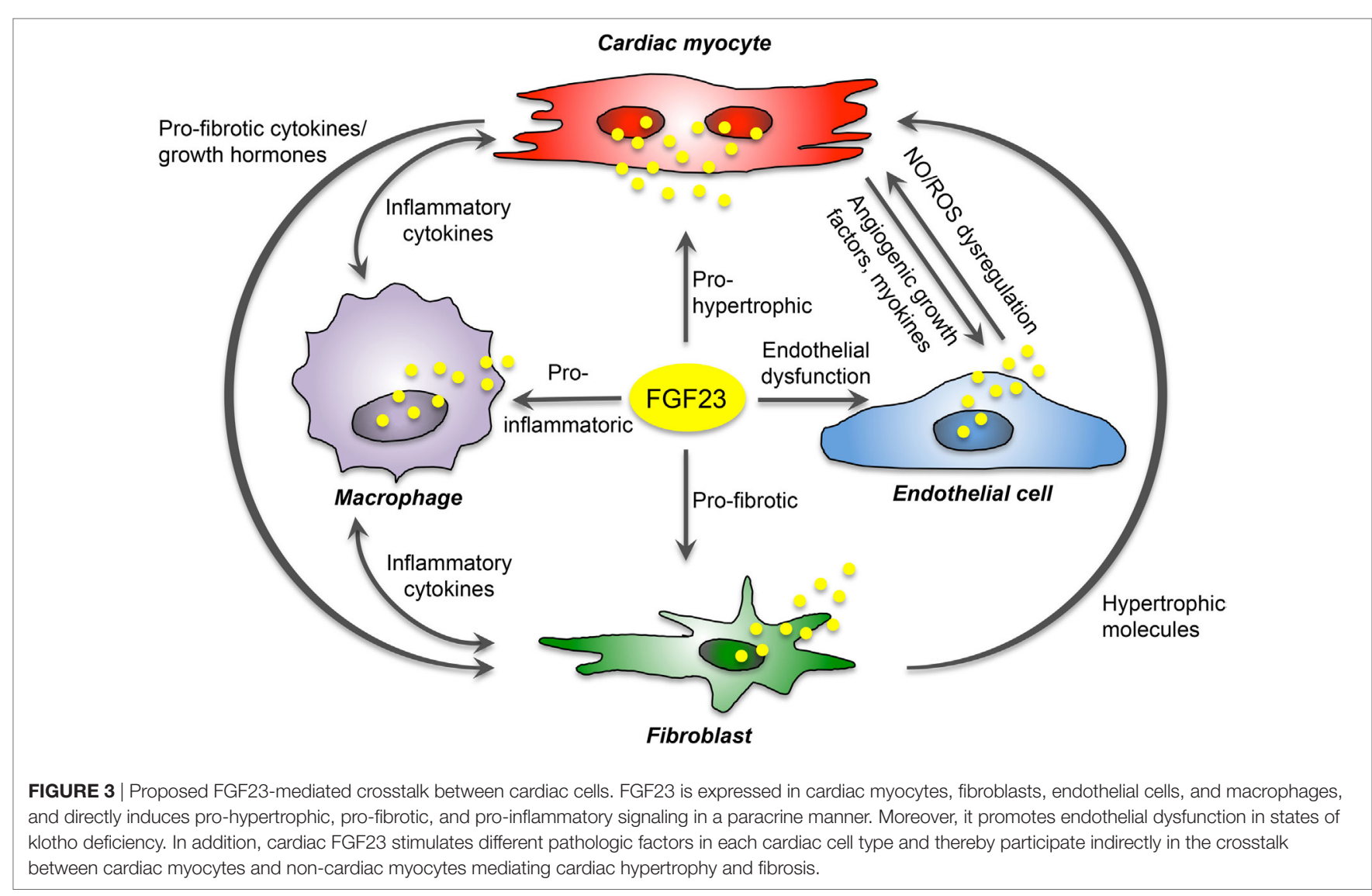


Another important point is that most clinical trials determine the circulating levels of C-term FGF23. What is about the intact FGF23 protein in HF patients, which is discussed to be more important to mediate signaling events, and do RAAS inhibitory therapeutics impact on the posttranslational modification and/ or cleavage of circulating and cardiac FGF23? It was shown in patients with autosomal dominant hypophosphatemic rickets, hyperphosphatemic familial tumoral calcinosis, and CKD, that the fraction of total FGF23 species representing intact biologically active hormone can markedly vary between different clinical settings (111). The cleavage of FGF23 in the bone is very well established, but less is known about the local processing of FGF23 in the heart and different cardiac cell types. The measurement of both biologically active and total FGF23 protein would represent an important advance for the understanding of its role also in different cardiovascular diseases. Moreover, future studies should provide more insights in the impact of therapeutic medications on the synthesis and cleavage of circulating and cardiac FGF23.

Important to note, treatment with $\mathrm{ACEi}$ or vitamin $\mathrm{D}$ was shown to improve soluble klotho levels in pediatric patients presenting with mild to moderate CKD (112). Taken together, these observations support the hypothesis of a direct cross talk between RAAS, vitamin D, FGF23, and klotho pathways, which can be targeted by RAAS inhibitor treatment. Normalization of soluble klotho may result in further modification of the specific FGFR for FGF23-mediated signaling in the heart though the interaction of FGF23 with klotho (65). It remains to be proven whether this will result in prevention of FGF23-driven pathological cardiac remodeling and counterregulate FGF23 cardiac toxicity.

\section{SUMMARY}

Enhanced cardiac FGF23 is reported in various clinical and experimental settings of cardiac remodeling or failure, e.g., CKD, ADHF, ischemic cardiomyopathy, myocarditis, dilated cardiomyopathy, inflammatory HF, TAC-induced pressure overload, experimental MI, and ischemia reperfusion in rodents. On a cellular level, FGF23 is expressed in cardiac myocytes and in other non-cardiac myocytes, including cardiac fibroblasts, vascular smooth muscle, and endothelial cells in coronary arteries, and in inflammatory macrophages (Figure 2).

The communication of cardiac myocytes and non-cardiac myocytes is mediated by intracellular signaling promoting pathologic ventricular remodeling and hypertrophy. Thereby, cardiac myocytes respond to stress stimuli with secretion of inflammatory cytokines, and damage-associated molecular patterns to impact on local non-cardiac myocytes. This activates resident macrophages and fibroblasts, which secrete pro-hypertrophic and pro-fibrotic cytokines to promote cardiac myocyte hypertrophy,

\section{REFERENCES}

1. Itoh N, Ornitz DM. Evolution of the Fgf and Fgfr gene families. Trends Genet (2004) 20:563-9. doi:10.1016/j.tig.2004.08.007

2. Itoh N, Ornitz DM. Fibroblast growth factors: from molecular evolution to roles in development, metabolism and disease. J Biochem (2011) 149:121-30. doi:10.1093/jb/mvq121 fibroblast differentiation, matrix deposition, and finally interstitial myocardial fibrosis. Current data suggest that secreted by cardiac myocytes, FGF23 can stimulate pro-fibrotic factors in myocytes to induce fibrosis-related pathways in fibroblasts and consequently cardiac fibrosis in a paracrine manner, and while acting on cardiac myocytes, FGF23 directly induces pro-hypertrophic genes and promotes the progression of LVH in an autocrine fashion. In addition, in vitro studies suggest that FGF23 can induce endothelial dysfunction in the setting of klotho deficiency via enhanced ROS production and suppression of antioxidant enzymes resulting in increased oxidative stress, which in turn may contribute to the progression of cardiovascular disease in humans. Finally, endogenous FGF23 expression and secretion in infiltrating macrophages in the heart induce cardiovascular disease progression through induction of inflammation and fibrosis. Overall, despite the known expression in bone, FGF23 is now established as also synthesized and secreted in the heart of humans and rodents by different cardiac cell types. Therefore, cardiac FGF23 is involved in the complex interaction between cardiac myocytes, fibroblasts, endothelial cell, and macrophages to induce signaling pathways and phenotypic changes within the same or nearby cells in an autocrine or paracrine manner, respectively (Figure 3).

Nevertheless, recent studies suggest that FGF23 is involved in the activation of local RAAS and, in turn, AngII and aldosterone as active RAAS components induce the expression of FGF23 in the heart in a feed-forward loop. It is known that RAAS activation promotes pro-inflammatory, pro-fibrotic, and pro-hypertrophic processes in cardiac cells. Since AT1R is present in cardiac myocytes, fibroblasts, and macrophages, these cell types are potent target cells for local, non-canonical RAAS activation and paracrine FGF23 signaling.

However, hard evidence showing that enhanced cardiac FGF23 directly induces pathologic cardiac remodeling and hypertrophy in a paracrine manner is lacking. The induction of cardiac FGF23 may also be a consequence of the failing heart. Whether cardiac FGF23 alone is sufficient to induce spontaneous cardiac hypertrophy and fibrosis and whether high cardiac synthesis of FGF23 always leads to the progression of HF must be investigated in further studies. Finally, which factor directly stimulates cardiac FGF23 expression and under which conditions cardiac FGF23 leads to pathological changes of the heart must also be clarified in suitable experimental studies and in specific animal models.

\section{AUTHOR CONTRIBUTIONS}

ML-N and DH equally contributed to conception and design of the manuscript; ML-N performed extensive literature search and wrote the first draft of the review; DH critically reviewed the manuscript. Both authors read and approved the final manuscript.

3. Ornitz DM, Itoh $\mathrm{N}$. The fibroblast growth factor signaling pathway. Wiley Interdiscip Rev Dev Biol (2015) 4:215-66. doi:10.1002/wdev.176

4. Brewer JR, Mazot P, Soriano P. Genetic insights into the mechanisms of Fgf signaling. Genes Dev (2016) 30:751-71. doi:10.1101/gad.277137.115

5. Itoh N, Ohta H, Nakayama Y, Konishi M. Roles of FGF signals in heart development, health, and disease. Front Cell Dev Biol (2016) 4:110. doi:10.3389/ fcell.2016.00110 
6. Spivak-Kroizman T, Lemmon MA, Dikic I, Ladbury JE, Pinchasi D, Huang J, et al. Heparin-induced oligomerization of FGF molecules is responsible for FGF receptor dimerization, activation, and cell proliferation. Cell (1994) 79:1015-24. doi:10.1016/0092-8674(94)90032-9

7. Mohammadi M, Olsen SK, Ibrahimi OA. Structural basis for fibroblast growth factor receptor activation. Cytokine Growth Factor Rev (2005) 16: 107-37. doi:10.1016/j.cytogfr.2005.01.008

8. Kurosu H, Ogawa Y, Miyoshi M, Yamamoto M, Nandi A, Rosenblatt KP, et al. Regulation of fibroblast growth factor-23 signaling by klotho. J Biol Chem (2006) 281:6120-3. doi:10.1074/jbc.C500457200

9. Urakawa I, Yamazaki Y, Shimada T, Iijima K, Hasegawa H, Okawa K, et al. Klotho converts canonical FGF receptor into a specific receptor for FGF23. Nature (2006) 444:770-4. doi:10.1038/nature05315

10. Goetz R, Beenken A, Ibrahimi OA, Kalinina J, Olsen SK, Eliseenkova AV, et al. Molecular insights into the klotho-dependent, endocrine mode of action of fibroblast growth factor 19 subfamily members. Mol Cell Biol (2007) 27:3417-28. doi:10.1128/MCB.02249-06

11. Goldfarb M, Schoorlemmer J, Williams A, Diwakar S, Wang Q, Huang X, et al. Fibroblast growth factor homologous factors control neuronal excitability through modulation of voltage-gated sodium channels. Neuron (2007) 55:449-63. doi:10.1016/j.neuron.2007.07.006

12. Fon Tacer K, Bookout AL, Ding X, Kurosu H, John GB, Wang L, et al. Research resource: comprehensive expression atlas of the fibroblast growth factor system in adult mouse. Mol Endocrinol (2010) 24:2050-64. doi:10.1210/ me.2010-0142

13. Itoh N, Ohta H. Pathophysiological roles of FGF signaling in the heart. Front Physiol (2013) 4:247. doi:10.3389/fphys.2013.00247

14. Zhang X, Ibrahimi OA, Olsen SK, Umemori H, Mohammadi M, Ornitz DM. Receptor specificity of the fibroblast growth factor family. The complete mammalian FGF family. J Biol Chem (2006) 281:15694-700. doi:10.1074/ jbc.M601252200

15. Goetz R, Mohammadi M. Exploring mechanisms of FGF signalling through the lens of structural biology. Nat Rev Mol Cell Biol (2013) 14:166-80. doi:10.1038/nrm3528

16. Carter EP, Fearon AE, Grose RP. Careless talk costs lives: fibroblast growth factor receptor signalling and the consequences of pathway malfunction. Trends Cell Biol (2015) 25:221-33. doi:10.1016/j.tcb.2014.11.003

17. Shimada T, Hasegawa H, Yamazaki Y, Muto T, Hino R, Takeuchi Y, et al. FGF23 is a potent regulator of vitamin D metabolism and phosphate homeostasis. J Bone Miner Res (2004) 19:429-35. doi:10.1359/JBMR.0301264

18. Kuro-o M, Matsumura Y, Aizawa H, Kawaguchi H, Suga T, Utsugi T, et al. Mutation of the mouse klotho gene leads to a syndrome resembling ageing. Nature (1997) 390:45-51. doi:10.1038/36285

19. Tagliabracci VS, Engel JL, Wiley SE, Xiao J, Gonzalez DJ, Nidumanda Appaiah $\mathrm{H}$, et al. Dynamic regulation of FGF23 by Fam20C phosphorylation, GalNAc-T3 glycosylation, and furin proteolysis. Proc Natl Acad Sci U S A (2014) 111:5520-5. doi:10.1073/pnas.1402218111

20. Gattineni J, Bates C, Twombley K, Dwarakanath V, Robinson ML, Goetz R, et al. FGF23 decreases renal $\mathrm{NaPi}-2 \mathrm{a}$ and $\mathrm{NaPi}-2 \mathrm{c}$ expression and induces hypophosphatemia in vivo predominantly via FGF receptor 1. Am J Physiol Renal Physiol (2009) 297:F282-91. doi:10.1152/ajprenal.90742.2008

21. Ben-Dov IZ, Galitzer H, Lavi-Moshayoff V, Goetz R, Kuro-O M, Mohammadi M, et al. The parathyroid is a target organ for FGF23 in rats. J Clin Invest (2007) 117:4003-8. doi:10.1172/JCI32409

22. Quarles LD. Skeletal secretion of FGF-23 regulates phosphate and vitamin D metabolism. Nat Rev Endocrinol (2012) 8:276-86. doi:10.1038/nrendo. 2011.218

23. Andrukhova O, Slavic S, Odorfer KI, Erben RG. Experimental myocardial infarction upregulates circulating fibroblast growth factor-23. J Bone Miner Res (2015) 30:1831-9. doi:10.1002/jbmr.2527

24. Hao H, Li X, Li Q, Lin H, Chen Z, Xie J, et al. FGF23 promotes myocardial fibrosis in mice through activation of beta-catenin. Oncotarget (2016) 7:64649-64. doi:10.18632/oncotarget.11623

25. Leifheit-Nestler M, Grosse Siemer R, Flasbart K, Richter B, Kirchhoff F, Ziegler WH, et al. Induction of cardiac FGF23/FGFR4 expression is associated with left ventricular hypertrophy in patients with chronic kidney disease. Nephrol Dial Transplant (2016) 31:1088-99. doi:10.1093/ndt/gfv421

26. Leifheit-Nestler M, Grabner A, Hermann L, Richter B, Schmitz K, Fischer DC, et al. Vitamin D treatment attenuates cardiac FGF23/FGFR4 signaling and hypertrophy in uremic rats. Nephrol Dial Transplant (2017) 32:1493-503. doi:10.1093/ndt/gfw454

27. Bienaime F, Ambolet A, Aussilhou B, Brazier F, Fouchard M, Viau A, et al. Hepatic production of fibroblast growth factor 23 in autosomal dominant polycystic kidney disease. J Clin Endocrinol Metab (2018). doi:10.1210/ jc.2018-00123

28. Zanchi C, Locatelli M, Benigni A, Corna D, Tomasoni S, Rottoli D, et al. Renal expression of FGF23 in progressive renal disease of diabetes and the effect of ACE inhibitor. PLoS One (2013) 8:e70775. doi:10.1371/journal. pone.0070775

29. Spichtig D, Zhang H, Mohebbi N, Pavik I, Petzold K, Stange G, et al. Renal expression of FGF23 and peripheral resistance to elevated FGF23 in rodent models of polycystic kidney disease. Kidney Int (2014) 85:1340-50. doi:10.1038/ki.2013.526

30. Mace ML, Gravesen E, Nordholm A, Hofman-Bang J, Secher T, Olgaard K, et al. Kidney fibroblast growth factor 23 does not contribute to elevation of its circulating levels in uremia. Kidney Int (2017) 92:165-78. doi:10.1016/j. kint.2017.01.015

31. Smith ER, Tan SJ, Holt SG, Hewitson TD. FGF23 is synthesised locally by renal tubules and activates injury-primed fibroblasts. Sci Rep (2017) 7:3345. doi:10.1038/s41598-017-02709-w

32. Sugiura H, Matsushita A, Futaya M, Teraoka A, Akiyama KI, Usui N, et al. Fibroblast growth factor 23 is upregulated in the kidney in a chronic kidney disease rat model. PLoS One (2018) 13:e0191706. doi:10.1371/journal. pone. 0191706

33. Himmelfarb J, Ikizler TA. Hemodialysis. N Engl J Med (2010) 363:1833-45. doi:10.1056/NEJMra0902710

34. Faul C, Amaral AP, Oskouei B, Hu MC, Sloan A, Isakova T, et al. FGF23 induces left ventricular hypertrophy. JClin Invest (2011) 121:4393-408. doi:10.1172/JCI46122

35. Gutierrez OM, Mannstadt M, Isakova T, Rauh-Hain JA, Tamez H, Shah A, et al. Fibroblast growth factor 23 and mortality among patients undergoing hemodialysis. NEnglJ Med (2008) 359:584-92. doi:10.1056/NEJMoa0706130

36. Touchberry CD, Green TM, Tchikrizov V, Mannix JE, Mao TF, Carney BW, et al. FGF23 is a novel regulator of intracellular calcium and cardiac contractility in addition to cardiac hypertrophy. Am J Physiol Endocrinol Metab (2013) 304:E863-73. doi:10.1152/ajpendo.00596.2012

37. Grabner A, Amaral AP, Schramm K, Singh S, Sloan A, Yanucil C, et al. Activation of cardiac fibroblast growth factor receptor 4 causes left ventricular hypertrophy. Cell Metab (2015) 22:1020-32. doi:10.1016/j.cmet.2015.09.002

38. Gutierrez OM, Januzzi JL, Isakova T, Laliberte K, Smith K, Collerone G, et al. Fibroblast growth factor 23 and left ventricular hypertrophy in chronic kidney disease. Circulation (2009) 119:2545-52. doi:10.1161/ CIRCULATIONAHA.108.844506

39. Nasrallah MM, El-Shehaby AR, Salem MM, Osman NA, El Sheikh E, Sharaf El Din UA. Fibroblast growth factor-23 (FGF-23) is independently correlated to aortic calcification in haemodialysis patients. Nephrol Dial Transplant (2010) 25:2679-85. doi:10.1093/ndt/gfq089

40. Yilmaz MI, Sonmez A, Saglam M, Yaman H, Kilic S, Demirkaya E, et al. FGF23 and vascular dysfunction in patients with stage 3 and 4 chronic kidney disease. Kidney Int (2010) 78:679-85. doi:10.1038/ki.2010.194

41. Seeherunvong W, Abitbol CL, Chandar J, Rusconi P, Zilleruelo GE, Freundlich M. Fibroblast growth factor 23 and left ventricular hypertrophy in children on dialysis. Pediatr Nephrol (2012) 27:2129-36. doi:10.1007/ s00467-012-2224-7

42. Scialla JJ, Lau WL, Reilly MP, Isakova T, Yang HY, Crouthamel MH, et al. Fibroblast growth factor 23 is not associated with and does not induce arterial calcification. Kidney Int (2013) 83:1159-68. doi:10.1038/ki.2013.3

43. Sarmento-Dias M, Santos-Araujo C, Poinhos R, Oliveira B, Silva IS, Silva LS, et al. Fibroblast growth factor 23 is associated with left ventricular hypertrophy, not with uremic vasculopathy in peritoneal dialysis patients. Clin Nephrol (2016) 85:135-41. doi:10.5414/CN108716

44. Baia LC, Humalda JK, Vervloet MG, Navis G, Bakker SJ, De Borst MH, et al. Fibroblast growth factor 23 and cardiovascular mortality after kidney transplantation. Clin J Am Soc Nephrol (2013) 8:1968-78. doi:10.2215/CJN 01880213

45. Hsu HJ, Wu MS. Fibroblast growth factor 23: a possible cause of left ventricular hypertrophy in hemodialysis patients. Am J Med Sci (2009) 337: 116-22. doi:10.1097/MAJ.0b013e3181815498 
46. Seiler S, Reichart B, Roth D, Seibert E, Fliser D, Heine GH. FGF-23 and future cardiovascular events in patients with chronic kidney disease before initiation of dialysis treatment. Nephrol Dial Transplant (2010) 25:3983-9. doi:10.1093/ndt/gfq309

47. Isakova $\mathrm{T}$, Xie $\mathrm{H}$, Yang $\mathrm{W}$, Xie $\mathrm{D}$, Anderson $\mathrm{AH}$, Scialla J, et al. Fibroblast growth factor 23 and risks of mortality and end-stage renal disease in patients with chronic kidney disease. JAMA (2011) 305:2432-9. doi:10.1001/ jama.2011.826

48. Kirkpantur A, Balci M, Gurbuz OA, Afsar B, Canbakan B, Akdemir R, et al. Serum fibroblast growth factor-23 (FGF-23) levels are independently associated with left ventricular mass and myocardial performance index in maintenance haemodialysis patients. Nephrol Dial Transplant (2011) 26:1346-54. doi:10.1093/ndt/gfq539

49. Mirza MA, Larsson A, Melhus H, Lind L, Larsson TE. Serum intact FGF23 associate with left ventricular mass, hypertrophy and geometry in an elderly population. Atherosclerosis (2009) 207:546-51. doi:10.1016/j. atherosclerosis.2009.05.013

50. Parker BD, Schurgers LJ, Brandenburg VM, Christenson RH, Vermeer C, Ketteler M, et al. The associations of fibroblast growth factor 23 and uncarboxylated matrix Gla protein with mortality in coronary artery disease: the Heart and Soul Study. Ann Intern Med (2010) 152:640-8. doi:10.7326/ 0003-4819-152-10-201005180-00004

51. Seiler S, Cremers B, Rebling NM, Hornof F, Jeken J, Kersting S, et al. The phosphatonin fibroblast growth factor 23 links calcium-phosphate metabolism with left-ventricular dysfunction and atrial fibrillation. Eur Heart J (2011) 32:2688-96. doi:10.1093/eurheartj/ehr215

52. Arnlov J, Carlsson AC, Sundstrom J, Ingelsson E, Larsson A, Lind L, et al. Higher fibroblast growth factor-23 increases the risk of all-cause and cardiovascular mortality in the community. Kidney Int (2013) 83:160-6. doi:10.1038/ki.2012.327

53. Isakova T, Houston J, Santacruz L, Schiavenato E, Somarriba G, Harmon WG, et al. Associations between fibroblast growth factor 23 and cardiac characteristics in pediatric heart failure. Pediatr Nephrol (2013) 28:2035-42. doi:10.1007/s00467-013-2515-7

54. Shibata K, Fujita S, Morita H, Okamoto Y, Sohmiya K, Hoshiga M, et al. Association between circulating fibroblast growth factor 23, alpha-klotho, and the left ventricular ejection fraction and left ventricular mass in cardiology inpatients. PLoS One (2013) 8:e73184. doi:10.1371/journal. pone. 0073184

55. Agarwal I, Ide N, Ix JH, Kestenbaum B, Lanske B, Schiller NB, et al. Fibroblast growth factor-23 and cardiac structure and function. J Am Heart Assoc (2014) 3:e000584. doi:10.1161/JAHA.113.000584

56. Imazu M, Takahama H, Asanuma H, Funada A, Sugano Y, Ohara T, et al. Pathophysiological impact of serum fibroblast growth factor 23 in patients with nonischemic cardiac disease and early chronic kidney disease. Am J Physiol Heart Circ Physiol (2014) 307:H1504-11. doi:10.1152/ajpheart.00331.2014

57. Kestenbaum B, Sachs MC, Hoofnagle AN, Siscovick DS, Ix JH, RobinsonCohen $\mathrm{C}$, et al. Fibroblast growth factor-23 and cardiovascular disease in the general population: the multi-ethnic study of atherosclerosis. Circ Heart Fail (2014) 7:409-17. doi:10.1161/CIRCHEARTFAILURE.113.000952

58. Wright CB, Dong C, Stark M, Silverberg S, Rundek T, Elkind MS, et al. Plasma FGF23 and the risk of stroke: the Northern Manhattan Study (NOMAS). Neurology (2014) 82:1700-6. doi:10.1212/WNL.0000000000000410

59. Wohlfahrt P, Melenovsky V, Kotrc M, Benes J, Jabor A, Franekova J, et al. Association of fibroblast growth factor-23 levels and angiotensin-converting enzyme inhibition in chronic systolic heart failure. JACC Heart Fail (2015) 3:829-39. doi:10.1016/j.jchf.2015.05.012

60. Andersen IA, Huntley BK, Sandberg SS, Heublein DM, Burnett JC Jr. Elevation of circulating but not myocardial FGF23 in human acute decompensated heart failure. Nephrol Dial Transplant (2016) 31:767-72. doi:10.1093/ndt/ gfv398

61. Wright CB, Shah NH, Mendez AJ, Derosa JT, Yoshita M, Elkind MS, et al. Fibroblast growth factor 23 is associated with subclinical cerebrovascular damage: the Northern Manhattan Study. Stroke (2016) 47:923-8. doi:10.1161/ STROKEAHA.115.012379

62. Faul C. Fibroblast growth factor 23 and the heart. Curr Opin Nephrol Hypertens (2012) 21:369-75. doi:10.1097/MNH.0b013e32835422c4

63. Kovesdy CP, Quarles LD. The role of fibroblast growth factor- 23 in cardiorenal syndrome. Nephron Clin Pract (2013) 123:194-201. doi:10.1159/000353593
64. Erben RG. Update on FGF23 and klotho signaling. Mol Cell Endocrinol (2016) 432:56-65. doi:10.1016/j.mce.2016.05.008

65. Grabner A, Faul C. The role of fibroblast growth factor 23 and klotho in uremic cardiomyopathy. Curr Opin Nephrol Hypertens (2016) 25:314-24. doi:10.1097/MNH.0000000000000231

66. Kovesdy CP, Quarles LD. FGF23 from bench to bedside. Am J Physiol Renal Physiol (2016) 310:F1168-74. doi:10.1152/ajprenal.00606.2015

67. Erben RG. Pleiotropic actions of FGF23. Toxicol Pathol (2017) 45:904-10. doi: $10.1177 / 0192623317737469$

68. Faul C. Cardiac actions of fibroblast growth factor 23. Bone (2017) 100:69-79. doi:10.1016/j.bone.2016.10.001

69. Haffner D, Leifheit-Nestler M. Extrarenal effects of FGF23. Pediatr Nephrol (2017) 32:753-65. doi:10.1007/s00467-016-3505-3

70. Heine GH, Seiler S, Fliser D. FGF-23: the rise of a novel cardiovascular risk marker in CKD. Nephrol Dial Transplant (2012) 27:3072-81. doi:10.1093/ ndt/gfs 259

71. Scialla JJ, Wolf M. Roles of phosphate and fibroblast growth factor 23 in cardiovascular disease. Nat Rev Nephrol (2014) 10:268-78. doi:10.1038/ nrneph.2014.49

72. Tanaka S, Fujita S, Kizawa S, Morita H, Ishizaka N. Association between FGF23, alpha-klotho, and cardiac abnormalities among patients with various chronic kidney disease stages. PLoS One (2016) 11:e0156860. doi:10.1371/ journal.pone.0156860

73. Shimada T, Mizutani S, Muto T, Yoneya T, Hino R, Takeda S, et al. Cloning and characterization of FGF23 as a causative factor of tumor-induced osteomalacia. Proc Natl Acad Sci U S A (2001) 98:6500-5. doi:10.1073/pnas. 101545198

74. Gheorghiade M, Zannad F, Sopko G, Klein L, Pina IL, Konstam MA, et al. Acute heart failure syndromes: current state and framework for future research. Circulation (2005) 112:3958-68. doi:10.1161/CIRCULATIONAHA. 105.590091

75. Slavic S, Ford K, Modert M, Becirovic A, Handschuh S, Baierl A, et al. Genetic ablation of Fgf 23 or klotho does not modulate experimental heart hypertrophy induced by pressure overload. Sci Rep (2017) 7:11298. doi:10.1038/ s41598-017-10140-4

76. Zhang B, Umbach AT, Chen H, Yan J, Fakhri H, Fajol A, et al. Up-regulation of FGF23 release by aldosterone. Biochem Biophys Res Commun (2016) 470:384-90. doi:10.1016/j.bbrc.2016.01.034

77. Kakkar R, Lee RT. Intramyocardial fibroblast myocyte communication. Circ Res (2010) 106:47-57. doi:10.1161/CIRCRESAHA.109.207456

78. Schultz JE, Witt SA, Nieman ML, Reiser PJ, Engle SJ, Zhou M, et al. Fibroblast growth factor-2 mediates pressure-induced hypertrophic response. J Clin Invest (1999) 104:709-19. doi:10.1172/JCI7315

79. Jiang ZS, Jeyaraman M, Wen GB, Fandrich RR, Dixon IM, Cattini PA, et al. High- but not low-molecular weight FGF-2 causes cardiac hypertrophy in vivo; possible involvement of cardiotrophin-1. J Mol Cell Cardiol (2007) 42:222-33. doi:10.1016/j.yjmcc.2006.09.002

80. Xiao L, Naganawa T, Lorenzo J, Carpenter TO, Coffin JD, Hurley MM. Nuclear isoforms of fibroblast growth factor 2 are novel inducers of hypophosphatemia via modulation of FGF23 and klotho. J Biol Chem (2010) 285:2834-46. doi:10.1074/jbc.M109.030577

81. Xiao L, Esliger A, Hurley MM. Nuclear fibroblast growth factor 2 (FGF2) isoforms inhibit bone marrow stromal cell mineralization through FGF23/ FGFR/MAPK in vitro. J Bone Miner Res (2013) 28:35-45. doi:10.1002/ jbmr.1721

82. Dai B, David V, Martin A, Huang J, Li H, Jiao Y, et al. A comparative transcriptome analysis identifying FGF23 regulated genes in the kidney of a mouse CKD model. PLoS One (2012) 7:e44161. doi:10.1371/journal.pone. 0044161

83. Zhou L, Li Y, Zhou D, Tan RJ, Liu Y. Loss of klotho contributes to kidney injury by derepression of Wnt/beta-catenin signaling. J Am Soc Nephrol (2013) 24:771-85. doi:10.1681/ASN.2012080865

84. Smith ER, Holt SG, Hewitson TD. FGF23 activates injury-primed renal fibroblasts via FGFR4-dependent signalling and enhancement of TGFbeta autoinduction. Int J Biochem Cell Biol (2017) 92:63-78. doi:10.1016/j. biocel.2017.09.009

85. Lam AP, Gottardi CJ. beta-catenin signaling: a novel mediator of fibrosis and potential therapeutic target. Curr Opin Rheumatol (2011) 23:562-7. doi:10.1097/BOR.0b013e32834b3309 
86. Guo Y, Xiao L, Sun L, Liu F. Wnt/beta-catenin signaling: a promising new target for fibrosis diseases. Physiol Res (2012) 61:337-46.

87. Leifheit-Nestler M, Kirchhoff F, Nespor J, Richter B, Soetje B, Klintschar M, et al. FGF23 is induced by activated renin-angiotensin-aldosterone system in cardiac myocytes and promotes the pro-fibrotic crosstalk between cardiac myocytes and fibroblasts. Nephrol Dial Transplant (2018). doi:10.1093/ndt/ gfy006

88. Mirza MA, Larsson A, Lind L, Larsson TE. Circulating fibroblast growth factor- 23 is associated with vascular dysfunction in the community. Atherosclerosis (2009) 205:385-90. doi:10.1016/j.atherosclerosis.2009.01.001

89. Voigt M, Fischer DC, Rimpau M, Schareck W, Haffner D. Fibroblast growth factor (FGF)-23 and fetuin-A in calcified carotid atheroma. Histopathology (2010) 56:775-88. doi:10.1111/j.1365-2559.2010.03547.x

90. van Venrooij NA, Pereira RC, Tintut Y, Fishbein MC, Tumber N, Demer LL, et al. FGF23 protein expression in coronary arteries is associated with impaired kidney function. Nephrol Dial Transplant (2014) 29:1525-32. doi:10.1093/ndt/gft523

91. Richter B, Haller J, Haffner D, Leifheit-Nestler M. Klotho modulates FGF23mediated NO synthesis and oxidative stress in human coronary artery endothelial cells. Pflugers Arch (2016) 468:1621-35. doi:10.1007/s00424-0161858-x

92. Frieler RA, Mortensen RM. Immune cell and other noncardiomyocyte regulation of cardiac hypertrophy and remodeling. Circulation (2015) 131: 1019-30. doi:10.1161/CIRCULATIONAHA.114.008788

93. MunozMendoza J, Isakova T, Ricardo AC, Xie H, Navaneethan SD, Anderson AH, et al. Fibroblast growth factor 23 and inflammation in CKD. Clin J Am Soc Nephrol (2012) 7:1155-62. doi:10.2215/CJN.13281211

94. Singh S, Grabner A, Yanucil C, Schramm K, Czaya B, Krick S, et al. Fibroblast growth factor 23 directly targets hepatocytes to promote inflammation in chronic kidney disease. Kidney Int (2016) 90:985-96. doi:10.1016/j.kint.2016. 05.019

95. Yan L, Bowman MA. Chronic sustained inflammation links to left ventricular hypertrophy and aortic valve sclerosis: a new link between S100/RAGE and FGF23. Inflamm Cell Signal (2014) 1:e279. doi:10.14800/ics.279

96. Dey G, Radhakrishnan A, Syed N, Thomas JK, Nadig A, Srikumar K, et al. Signaling network of oncostatin M pathway. J Cell Commun Signal (2013) 7:103-8. doi:10.1007/s12079-012-0186-y

97. Kubin T, Poling J, Kostin S, Gajawada P, Hein S, Rees W, et al. Oncostatin M is a major mediator of cardiomyocyte dedifferentiation and remodeling. Cell Stem Cell (2011) 9:420-32. doi:10.1016/j.stem.2011.08.013

98. Schaefer LK, Wang S, Schaefer TS. Oncostatin M activates stat DNA binding and transcriptional activity in primary human fetal astrocytes: low- and high-passage cells have distinct patterns of stat activation. Cytokine (2000) 12:1647-55. doi:10.1006/cyto.2000.0774

99. Van Wagoner NJ, Choi C, Repovic P, Benveniste EN. Oncostatin M regulation of interleukin-6 expression in astrocytes: biphasic regulation involving the mitogen-activated protein kinases ERK1/2 and p38. J Neurochem (2000) 75:563-75. doi:10.1046/j.1471-4159.2000.0750563.x

100. Arita K, South AP, Hans-Filho G, Sakuma TH, Lai-Cheong J, Clements S, et al. Oncostatin M receptor-beta mutations underlie familial primary localized cutaneous amyloidosis. Am J Hum Genet (2008) 82:73-80. doi:10.1016/j. ajhg.2007.09.002

101. Richter M, Polyakova V, Gajawada P, Pöling J, Warnecke H, Braun T, et al. Oncostatin M induces FGF23 expression in cardiomyocytes. JClin Exp Cardiolog (2012) S9:003. doi:10.4172/2155-9880.S9-003
102. Richter M, Lautze HJ, Walther T, Braun T, Kostin S, Kubin T. The failing heart is a major source of circulating FGF23 via oncostatin M receptor activation. J Heart Lung Transplant (2015) 34:1211-4. doi:10.1016/j.healun.2015. 06.007

103. Han X, Li L, Yang J, King G, Xiao Z, Quarles LD. Counter-regulatory paracrine actions of FGF-23 and 1,25(OH)2 D in macrophages. FEBS Lett (2016) 590:53-67. doi:10.1002/1873-3468.12040

104. Fajol A, Chen H, Umbach AT, Quarles LD, Lang F, Foller M. Enhanced FGF23 production in mice expressing PI3K-insensitive GSK3 is normalized by beta-blocker treatment. FASEB J (2016) 30:994-1001. doi:10.1096/f.15279943

105. Shalhoub V, Shatzen EM, Ward SC, Davis J, Stevens J, Bi V, et al. FGF23 neutralization improves chronic kidney disease-associated hyperparathyroidism yet increases mortality. J Clin Invest (2012) 122:2543-53. doi:10.1172/ JCI61405

106. Fukumoto S. Anti-fibroblast growth factor 23 antibody therapy. Curr Opin Nephrol Hypertens (2014) 23:346-51. doi:10.1097/01.mnh.0000447012. 98357.da

107. Katoh M. Therapeutics targeting FGF signaling network in human diseases. Trends Pharmacol Sci (2016) 37:1081-96. doi:10.1016/j.tips.2016.10.003

108. Udell JA, Morrow DA, Jarolim P, Sloan S, Hoffman EB, O'donnell TF, et al. Fibroblast growth factor-23, cardiovascular prognosis, and benefit of angiotensin-converting enzyme inhibition in stable ischemic heart disease. J Am Coll Cardiol (2014) 63:2421-8. doi:10.1016/j.jacc.2014.03.026

109. Ter Maaten JM, Voors AA, Damman K, Van Der Meer P, Anker SD, Cleland JG, et al. Fibroblast growth factor 23 is related to profiles indicating volume overload, poor therapy optimization and prognosis in patients with newonset and worsening heart failure. Int J Cardiol (2018) 253:84-90. doi:10.1016/j. ijcard.2017.10.010

110. Humalda JK, Lambers Heerspink HJ, Kwakernaak AJ, Slagman MC, Waanders F, Vervloet MG, et al. Fibroblast growth factor 23 and the antiproteinuric response to dietary sodium restriction during renin-angiotensinaldosterone system blockade. Am J Kidney Dis (2015) 65:259-66. doi:10.1053/j. ajkd.2014.07.022

111. Wolf M, White KE. Coupling fibroblast growth factor 23 production and cleavage: iron deficiency, rickets, and kidney disease. Curr Opin Nephrol Hypertens (2014) 23:411-9. doi:10.1097/01.mnh.0000447020.74593.6f

112. Shroff R, Aitkenhead H, Costa N, Trivelli A, Litwin M, Picca S, et al. Normal 25-hydroxyvitamin D levels are associated with less proteinuria and attenuate renal failure progression in children with CKD. J Am Soc Nephrol (2016) 27:314-22. doi:10.1681/ASN.2014090947

Conflict of Interest Statement: ML-N was supported by Amgen and received a research grant from the German Cardiac Society. DH received consultancy fees and/or research grants from Amgen, Chiesi Farmaceutici, Horizon Pharma, Kyowa Kirin, Pfizer, and Sandoz.

Copyright $\odot 2018$ Leifheit-Nestler and Haffner. This is an open-access article distributed under the terms of the Creative Commons Attribution License (CC BY). The use, distribution or reproduction in other forums is permitted, provided the original author(s) and the copyright owner are credited and that the original publication in this journal is cited, in accordance with accepted academic practice. No use, distribution or reproduction is permitted which does not comply with these terms. 